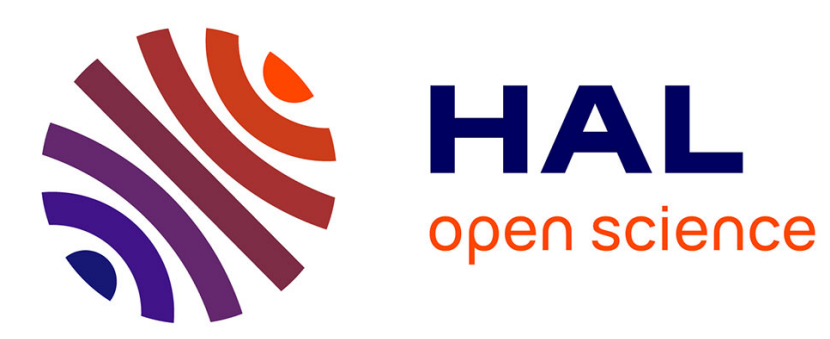

\title{
Des pôles aux laboratoires: les échelles de la coopération internationale en paléoclimatologie (1955-2015)
}

\author{
Morgan Jouvenet
}

\section{To cite this version:}

Morgan Jouvenet. Des pôles aux laboratoires: les échelles de la coopération internationale en paléoclimatologie (1955-2015). Revue française de sociologie, 2016. hal-02003621

\section{HAL Id: hal-02003621 \\ https://hal.science/hal-02003621}

Submitted on 14 Feb 2019

HAL is a multi-disciplinary open access archive for the deposit and dissemination of scientific research documents, whether they are published or not. The documents may come from teaching and research institutions in France or abroad, or from public or private research centers.
L'archive ouverte pluridisciplinaire HAL, est destinée au dépôt et à la diffusion de documents scientifiques de niveau recherche, publiés ou non, émanant des établissements d'enseignement et de recherche français ou étrangers, des laboratoires publics ou privés. 


\title{
Des pôles aux laboratoires : les échelles de la coopération internationale en paléoclimatologie (1955-2015)
}

\author{
Morgan JOUVENET
}

\begin{abstract}
Résumé. L'ice core science (ICS) est une spécialité de la paléoclimatologie permettant de reconstituer la dynamique des climats du passé, à partir de l'analyse en laboratoire de morceaux de glace forés au Groenland et en Antarctique. Les particularités du terrain polaire et les enjeux géopolitiques associés à ces territoires expliquent qu'elle se caractérise, dès son émergence, par une forte coopération internationale. Cet article rend compte du sens et des formes de cette coopération, en s'intéressant à l'organisation des campagnes de terrain par les scientifiques, et en présentant leur coordination au sein du réseau de mobilisation des fragiles archives climatiques - une infrastructure de globalisation reliant les pôles aux laboratoires. L'analyse du développement de l'ıcs révèle l'influence initiale du contexte de la Guerre froide sur la coopération scientifique internationale. Elle montre également comment cette spécialité a pérennisé son activité au-delà de ce contexte, en transformant la nature des pôles et en contribuant aux débats sur le réchauffement climatique. L'éclatement du « fait international », dans ce domaine, constitue un point d'appui empirique qui nourrit la réflexion sur le traitement du contexte dans les science studies, et la conclusion de l'article apporte des éléments en ce sens.
\end{abstract}

Mots-clés. Études des sciences - PalÉoclimatologie - CoOpération - Études TRANSNATIONALES - GLOBALISATION

Plusieurs observateurs (e.g. Ravetz, 2006 ; Pestre, 2010) ont associé la prégnance de la question environnementale, depuis les années 1970, à une évolution marquante de la place des sciences en société. La climatologie, en particulier, témoigne de l'importance prise par les sciences de l'environnement - des sciences de l'observation et de la complexité dont les résultats sont régulièrement commentés dans l'espace public. La composante paléoclimatique de ces résultats est fondamentale, dans la mesure où elle permet d'inscrire la question du changement climatique dans la longue durée, nourrissant la compréhension de ses paramètres déterminants et les projections sur ses probables évolutions. L'une de ses spécialités phares, l' «ice core science » (ICS), permet, depuis les années 1960, de saisir les rythmes et formes des variations climatiques, et de préciser le rôle des gaz à effet de serre dans ces phénomènes. Elle

Ce texte a bénéficié de mes échanges avec Michel Dubois, Yves Gingras et Claude Rosental, les coordinateurs du dossier "Internationalisation de la recherche scientifique » du présent numéro de la Revue française de sociologie. Les remarques des évaluateurs anonymes m'ont aussi permis de l'améliorer. Je tiens donc à tous les remercier pour leurs lectures, ainsi que Nicolas Dodier, avec qui j'ai pu en discuter lors de son écriture. Ma gratitude va également à toutes les personnes interrogées pour l'enquête exploitée ici, et en particulier à ceux qui, en plus de m'avoir accueilli dans leurs laboratoires, m'ont permis de participer à leurs activités ou donné accès à de précieux documents. 
est pourtant largement absente du corpus des science \& technology studies (STS), qui ont encore très peu éclairé la façon dont les connaissances sur les climats du passé sont produites : c'est peu dire en effet que, contrairement aux sciences de l'atmosphère et à l'océanologie, « la glaciologie et la paléoclimatologie demeurent sousétudiées ${ }^{1}$. Les chercheurs de l'ICS remontent le temps, jusqu'à un million d'années, en examinant des échantillons de glace polaire prélevés à 3000 mètres sous la surface (en Antarctique), avec une résolution qui (au Groenland) peut être saisonnière. La qualité des données ainsi produites (par analyse de la composition chimique de la glace et de l'air qu'elle contient) dépend aussi bien des «manips » réalisées en laboratoire que d'une pratique du terrain très structurante pour la communauté ICS. De ce point de vue, c'est un domaine bipolaire, non parce que ses objets proviennent des deux pôles, mais parce qu'il est fondé sur un dispositif assurant la circulation des chercheurs et de leurs objets entre le terrain et le laboratoire.

Ce dispositif est international, et donne toute sa visibilité au travail de coordination mené par-delà les frontières dès l'émergence de cette communauté. Cet article vise donc à saisir les formes, sens et enjeux de cette constante « internationalité » (et leur évolution) plutôt qu'à analyser un cas d' «internationalisation » progressive d'un domaine scientifique. Plus précisément, son but est de répondre à une double interrogation, portant à la fois sur les spécificités de l'objet (de fragiles échantillons de glace polaire) au cœur de ce domaine scientifique, et sur celles de sa période de développement (qui voit la géopolitique des régions polaires sensiblement évoluer et la question climatique s'affirmer) : quels rapports de nature sociologique peut-on établir entre ces spécificités et les formes de la coopération scientifique dans l'ICS ?

«Par définition, écrit l'un de ses acteurs, la recherche en milieux polaires [...] est menée dans un cadre totalement internationalisé. » (Jugie, 2007, p. 44). Les propos des chercheurs de l'ICS vont dans le même sens : malgré sa petit taille, estimée à 200 personnes, « la première caractéristique de cette communauté, c'est qu'elle est très internationale » $(\mathrm{t}-\mathrm{NM})^{2}$; « c'est véritablement une science internationale, c'est clair » (c-GL). Les avancées scientifiques marquantes de l'ICS sont dès lors présentées comme le fruit de programmes internationaux de grande envergure, organisés sur le mode de la «big science» (Galison et Hevly, 1992) ${ }^{3}$, et coordonnant le déploiement des moyens sur le terrain, la distribution des échantillons issus des forages (les «ice cores »[IC]), et la division du travail expérimental. Le ratio de copublications internationales est élevé (voir, par exemple, Jouzel, 2013), et certains articles fameux ont été signés par des consortium comme l'European Project for Ice Coring in Antarctica (EPICA), qui a rassemblé des chercheurs et techniciens issus d'une dizaine de pays (et a reçu, en 2009, le prix Descartes pour la science décerné par l'Union européenne). Les drapeaux et blasons qui ornent les spectaculaires images de ces campagnes polaires illustrent cette dimension internationale ; ils rappellent aussi que les scientifiques sont soutenus par des institutions nationales qui peuvent avoir des raisons très diverses de soutenir et de valoriser leur présence aux pôles. Dès lors, si le processus d'internationalisation souvent mis en avant pour caractériser le développement de

1. Comme l'indique Robert E. Kohler (2011, p. 218) dans un panorama de l'historiographie des field sciences.

2. Les extraits d'entretiens avec des chercheurs sont suivis de la mention c-XX, avec les techniciens de la mention t-YY. XX et YY sont des initiales attribuées aléatoirement à un individu.

3. L'institutionnalisation de son dispositif de coopération internationale, après la Seconde Guerre mondiale, incitait déjà Stephen J. Pyne à décrire la glaciologie polaire comme une «big science », avant même que sa branche paléoclimatique ne s'affirme ([1987] 2004, p. 284). Aant Elzinga (1993, p. 102) fait le même rapprochement. 
l'ICS peut être décrit à travers la mise en place d'un dispositif (associant acteurs, institutions et instruments) dont l'étendue et l'efficacité se sont accrues avec l'affirmation de la problématique du changement climatique - à l'instar de «l'immense appareillage » [vast machine] analysant l'atmosphère terrestre (Edwards, 2010) -, on peut aussi faire l'hypothèse que ces étendards ne sont pas insignifiants, et penser qu'ils invitent à ne pas confondre la " globalisation ${ }^{4}$ avec un effacement des différences nationales. La référence à la "globalisation» cache en effet souvent des « réseaux de relations transnationales d'inclusion (et d'exclusion) » susceptibles de révéler «des nœuds et des blocages autant que du mouvement; de la régulation et du contrôle autant que de la circulation et de la fluidité » (Krige, 2014b, p. 229-230, p. 244) $)^{5}$ on verra notamment ce que l'histoire de l'ICS doit aux enjeux géopolitiques attachés à l'Antarctique - et à leur «sublimation » (Elzinga, 1993, p. 87) dans le statut de réserve scientifique que lui a donné un traité signé en 1959.

L'étendue des dispositifs de coordination et de circulation qui soutiennent l'activité des paléoclimatologues de l'ICS incite ainsi à mener une "étude transnationale des sciences » centrée sur des «flux de personnes, biens, idées ou procédés » (Turchetti et al., 2012, p. 320), et susceptible de révéler comment - i.e. par quelles actions et dans quelles conditions - des acteurs " peuvent transformer des mondes à travers les connexions qu'ils établissent» dans des espaces frontières (Pestre, 2012, p. 429-430). L'étude de la dynamique transcontinentale de l'ICS montre que ces conditions sont à la fois naturelles et sociohistoriques, puisque les pratiques des scientifiques dépendent aussi bien des propriétés du terrain polaire (et de ses échantillons) que des ressources qui leur sont attribuées par des agences nationales ou internationales. Mais elle illustre également la façon dont, en retour, une spécialité scientifique peut gagner en crédit et influencer les relations internationales (Weiss, 2015) en contribuant à changer (la perception de) la nature. Pour analyser cette dynamique, j'examinerai trois niveaux d'activité déterminants pour le domaine ICS : 1) celui de la coopération entre scientifiques, sur le terrain et dans les réunions qui préparent les campagnes de forage ;2) celui du dispositif de partage des échantillons de glace polaire, qui assure leur circulation des pôles aux laboratoires et entre laboratoires ; 3) celui des relations internationales centrées sur ces territoires, qui modulent l'occupation du terrain scientifique suivant des logiques politiques et stratégiques. Il ne peut être question ici de rendre compte en détail de la texture de chacun de ces niveaux. L'éclatement du « fait international » caractéristique de l'ICS permet toutefois de nourrir une réflexion sur le contexte de la pratique scientifique, sur la façon dont on peut articuler différents sites et niveaux d'action pour saisir le développement d'un domaine scientifique, et je présenterai en conclusion des éléments de cette réflexion.

4. Chez Paul Edwards, la globalisation opérée par cette «machine » décrit aussi bien l'extension et l'intensification de la coopération internationale que l'amélioration de la couverture des données scientifiques.

5. De même, les études rassemblées dans Grégoire Mallard, Catherine Paradeise et Ashveen Peerbaye (2009, p. 31) «mettent en lumière les défis et les sources de conflit entre les différentes formes d'autorité politique qu'entraîne la mondialisation de la science ». 


\section{ENCADRÉ 1. - Méthode de l'enquête}

Le matériau empirique exploité ici est issu d'une enquête en cours sur l'histoire et la sociologie de l'ICS en France. Cette enquête s'appuie sur un recueil de données combinant plusieurs approches :

- Une série d'entretiens (de deux heures en moyenne) avec les chercheurs et techniciens de ce domaine $(n=28)$, hommes et femmes des trois générations (de la jeune docteure au DR CNRS émérite), membres des trois laboratoires travaillant sur les IC à Saclay, Grenoble et Aix-en-Provence.

- Une série d'entretiens (d'une heure et demie en moyenne) avec des climatologues (hors domaine ICS) et des acteurs des institutions de la recherche française $(\mathrm{n}=13)$.

- Des observations ethnographiques réalisées dans et autour des laboratoires : suivis de manips sur les dispositifs expérimentaux, discussions autour des instruments et ateliers, participation à une session de découpage d'IC dans l'entrepôt de stockage français, partage de moments formels (réunions de projets, soutenances, etc.) et informels (café, cantine, trajets, etc.) de la vie de laboratoire.

- Le dépouillement d'archives publiques et privées (1955-2015) : archives de la DGRST et du ministère de la Recherche (rapports et évaluations, documents de prospective, courriers, etc., concernant la politique de recherche en climatologie), archives de laboratoire (bilans pluriannuels, rapports de mission, présentations de manifestations scientifiques, etc.) et des chercheurs (courriers, journaux, brouillons de publication, etc. $)^{6}$.

- L'analyse (qualitative et quantitative) de documents : littérature scientifique et de vulgarisation, communications des organismes de recherche, films amateurs et professionnels, articles de presse.

\section{La coopération internationale sur le terrain}

Si le travail sur les IC réalisé en laboratoire apparait clairement dans les articles publiés dans Nature, Science, Climate of the Past, Journal of Geophysical Research, Journal of Glaciology, etc., l'évocation du terrain y est minimale, exprimée le plus souvent à travers les coordonnées spatiales (lieu, profondeur) et temporelles (date de l'extraction, âge de la glace) des échantillons utilisés. Sa pratique relève des savoirfaire tacites et du savoir implicite que l'écriture scientifique rend largement invisible. En revanche, dans les entretiens avec les chercheurs et les mémoires qu'ils publient (Lorius, 1993 ; Jouzel et al., 2008 ; Lorius et Carpentier, 2010 ; Petit, 2013 ; Alley [2000] 2014; Mayewski et White, 2002), les activités menées dans et autour des bases polaires, entre «landscape » et «labscape » (Kohler, 2002), occupent une place de choix. Ces récits restituent l'impact des particularités naturelles de leur terrain sur la culture des scientifiques. Ils montrent en effet combien les séjours sur la glace marquent les esprits et l'identité collective de la communauté ICS, en raison des spécificités des espaces naturels pratiqués, mais aussi des conditions de vie collective que celles-ci imposent à des équipes qui sont presque toujours internationales. Les récits de «l'aventure humaine » du terrain constituent dès lors également un révélateur d'une expérience de la dimension internationale de l'ICS à la fois très concrète et fondamentale pour ses chercheurs. Je vais donc commencer par livrer des éléments

6. NB : Seules les archives privées d'un chercheur, rassemblant des documents de travail concernant des coopérations internationales, ont été directement mobilisées pour cet article. 
d'une sociologie de la «dramaturgie du travail» (au sens de Hughes, 1976) sur le terrain, permettant de restituer cette expérience à partir de ces récits ${ }^{7}$.

\section{La Cité scientifique sur la glace : solidarité et science " extrême "}

Au-delà des gestes relativement simples qu'ils ont à accomplir lors des forages, les séjours sur le terrain ont des vertus cognitives évidentes pour les chercheurs ( on comprend des choses rien qu'à être sur le terrain et à regarder le terrain », c-DW). Ils constituent également des moments de socialisation à une culture et à une histoire spécifiques (Martin Rudwick, 1996, évoque des « rites de passage » pour les géologues), transmises de génération en génération lors d'interactions se déroulant dans des bases ou campements isolés. La singularité et la force de ces séjours, pour les chercheurs qui voyagent au Groenland et en Antarctique ${ }^{8}$, s'explique à la fois par les conditions de vie collective sur place et la rudesse du climat local - les premières étant largement fonction de la seconde. Leurs récits soulignent ainsi ces "affinités particulières qui proviennent des occupations, des privations et périls communs » (suivant une formule utilisée par Herman Melville au sujet des baleiniers, dans Moby Dick), et doivent, à ce titre, être pris en compte par l'histoire des sciences de terrain (Vetter, 2011, p. 9).

L'isolement des terrains polaires est considéré comme un modèle de mise à l'épreuve des capacités d'acclimatation et de coopération des individus (Grevsmühl, 2016). Pour les chercheurs de l'ICS, cette expérience se double d'une confrontation avec des cultures nationales différentes, auxquelles le relatif dénuement de la vie sur place donne une visibilité importante. Les comparaisons internationales émaillent ainsi les récits de l'organisation des activités « domestiques » du camp et des moments festifs. L'étrangeté de la consommation d'alcool' ${ }^{9}$, le goût pour le sauna ou les dispositions musicales des soviétiques ont ainsi fourni matière à de nombreuses considérations sur la diversité culturelle de la population des chercheurs et techniciens polaires. La vie sur place est animée par la découverte des habitudes culinaires ou sanitaires nationales ${ }^{10}$, dans un esprit parfois potache défiant l'ennui, voire le froid (pour cela, on peut "faire le tour du monde » dès la sortie du sauna). "C'est un partage culturel [...] C'est un peu le côté génial de la mondialisation. » (c-EZ). Dans cette perspective, la coopération internationale donne du relief à la vie sur la glace, sous une apparence qui peut être anecdotique mais constitue finalement l'un des incontournables aspects du travail sur le terrain.

7. Comme dans l'«anthropologie interprétative» de Sharon Traweek (1992, p. 101), je vise ici le sens donné par les scientifiques à leurs activités et interactions. Pour S. Traweek, il s'agit essentiellement de révéler les différences culturelles nationales qui s'expriment autour des instruments de la big science (1988).

8. On peut aussi extraire des $I C$ sur les glaciers des montagnes, mais les informations recueillies n'offrent pas le même intérêt pour la paléoclimatologie, du fait notamment de leur moindre profondeur et de leurs mouvements plus prononcés.

9. À la fois marqueur de différences et opérateur de rapprochement, l'alcool occupe une place à part dans ce folklore longtemps marqué par la figure du viril aventurier.

10. «Avec les Italiens, tu sais que tu mangeras très, très bien [...] les Anglais, ils ont une vision complètement différente de ça. [...] Pour eux, ça doit être un truc à la Shackleton. Un truc de guerrier [...] les Italiens, ils étaient tout heureux d'avoir une douche tous les jours. Les Anglais, une douche par semaine, ils étaient aux anges ! [...] c'était marrant de voir ces différences de culture. » (t-RR). «Le Français, il va plus dans le froid, il est plus roots... L'Italien, il aime bien ses pantoufles [...] il aime un peu moins le contact brutal avec la nature. » (c-DW). 
Les récits du terrain valent cependant également pour la mise en scène de l'effacement des différences (internationales, mais aussi hiérarchiques et statutaires : les conditions difficiles « lissent la hiérarchie », c-BS), dans la synergie collective de l'exploration des sites et de l'extraction des matériaux scientifiques. L'évocation de la concentration quasi exclusive sur le travail en groupe, et de la coupure avec les tracas du quotidien domestique et professionnel justifie la comparaison avec une douce utopie : «C'est une vie qui est relativement simple : on se lève, on va travailler, on mange, on se couche. C'est d'une simplicité monacale. » (t-MI) ; «C'est vraiment agréable... tu penses pas aux clefs, tu penses pas à ton code de carte bleue. » (t-RR) ; «C'est presque une forme utopique de société... un mode de vie idéal. » (c-HV). Les chercheurs et techniciens insistent aussi sur la force des rapprochements et convergences entre résidents, qui donnent à la base polaire les atours d'une Cité ${ }^{11}$ cosmopolite et scientifique idéale. Narrant son retour en Pennsylvanie après une longue campagne de forage, un scientifique étatsunien se demande ainsi " pourquoi le "monde réel" ne ressemble pas plus » à la base qu'il vient de quitter (Alley, [2000] 2014, p. 30). De même, l'un des chercheurs interrogés pour l'enquête raconte qu' « après 9 semaines [sur le terrain], quand on s'est retrouvés dans le métro à $5 \mathrm{~h}$ de l'après-midi, je me suis dit "mais qu'est-ce qu'on fait là ? On est complètement tarés" » (t-NM).

Peut-être que « la solidarité entre polaires permet plus facilement qu'ailleurs de se mettre d'accord », se demande l'un des pionniers de l'ICS (Lorius et Carpentier, 2010, p. 49). Ses propos et ceux de ses collègues indiquent que cette solidarité a des déterminants naturels et culturels, évoquant à la fois la cohésion nécessaire à la vie en milieu extrême et le partage d'une mission scientifique de connaissance du climat. Le slogan de Richard Byrd, explorateur étatsunien, est connu : le froid force l'union de tous (cité dans Pyne, [1987] 2004, p. 351), et les récits des accidents sur le terrain (chutes dans des crevasses, incendies sur les bases, crashs d'avions... « Donc en gros, l'avion a explosé sous mes yeux... », c-LM) incitent en effet à faire figurer l'ICS parmi les «sciences extrêmes »-comme il y a des «sports extrêmes »- à la pratique particulièrement dangereuse (Kohler, 2011, p. 229) ${ }^{12}$. Si les chercheurs en activité aujourd'hui soulignent le relatif confort des bases et équipement actuels, par rapport à ceux des deux premières générations de leurs collègues, l'isolement et le froid, ainsi que la nécessité de s'éloigner des bases pour rechercher de nouveaux sites de forage, inscrivent toujours l'ICS dans le lignage historique des héros de l'exploration polaire. Pour Claude Lorius, « [la] division entre aventure et science n'a pas lieu d'être, elles sont les deux visages d'une même curiosité humaine » (Lorius et Carpentier, 2010, p. 30), et la figure du «saventurier» (Étienne dans Hauglustaine et al., 2008, p. 6) est encore prégnante dans la culture de ce domaine scientifique.

C'est bien toutefois une mission scientifique qui rassemble aujourd'hui des hommes et (de plus en plus) des femmes sur ces terrains, et le souci de cette mission constitue un autre pilier de la Cité cosmopolite polaire. Ce souci se traduit de manière très évidente et concrète dans les efforts collectifs réalisés pour mener les forages à bien, préparer soigneusement les échantillons (découpage, étiquetage, etc.) pour leurs futures analyses en laboratoire, voire commencer ce travail d'analyse avec les

11. J'emploie ce terme pour souligner le caractère mythologique de cette représentation d'une « communauté politique indépendante » (voir le Trésor de la langue française, article « Cité », I) qui associe la proximité spatiale et la simplicité des échanges sur l'organisation de la vie collective.

12. «Sur la frontière antarctique, le triomphe et la mort sont d'inséparables compagnons », lit-on en exergue de l'un des premiers ouvrages consacrés aux sciences en Antarctique, dédié à la mémoire d'un scientifique disparu sur le terrain (Lewis, 1965). 
instruments (de plus en plus) emmenés sur place. Un exemple de coopération (ou de « coopétition » ?) marquant est donné par les équipes de foreurs, qui, s'ils se livrent une concurrence internationale aussi ludique qu'acharnée (« Nous, ce qui nous intéresse à la fin de la journée, c'est combien de mètres on a fait [...] Même si on s'entend tous bien, on est toujours bien content d'avoir fait 5 mètres de plus à la fin de la journée que son copain. », t-MI), ne rechignent pas à s'entraider en cas de panne et de blocage. L'histoire de l'ICS est marquée par de tels épisodes de solidarité scientifique scellée sur le terrain, proposant un contraste fort avec un monde « extérieur» défiguré par les frontières nationales. Il parait évident que le partage des conditions de vie difficiles et la découverte d'objectifs scientifiques communs, dans le cadre d'une spécialité encore émergente, a en effet permis de «tisser des liens très forts, en particulier pour ceux qui ont [eu] la chance de se retrouver sur le terrain » (Jouzel et al., 2008, p. 109). Et le contraste était certainement à son maximum lors de la Guerre froide, lorsque des chercheurs américains, français et soviétiques ont pu profiter du cadre du Traité de l'Antarctique, pour développer « une coopération internationale dans un lieu sans patrie» (Lorius et Carpentier, 2010, p. 162). Le terrain apparaît alors comme le foyer d'une communauté capable d'atténuer, sinon de protéger, ses membres des accidents politiques de l'histoire. Ainsi, les liens noués en Antarctique entre chercheurs français et soviétiques ${ }^{13}$ expliquent que, lors de l'effondrement de l'URSS, en 1991, les premiers se soient mobilisés pour trouver des financements permettant aux seconds de poursuivre leurs activités (comme le montrent plusieurs documents d'archives).

On trouve déjà les prémisses de la rhétorique de la Cité scientifique internationale dans la pratique du «terrain » des savants voyageurs du XVIII siècle (Sörlin, 1993, p. 67). Chez les premiers explorateurs des glaces, notamment, elle permettait de contrebalancer une autre rhétorique, celle de la rivalité nationale de la course aux pôles (ibid.). Cette oscillation a perduré chez des scientifiques qui ont suivi leurs traces au $\mathrm{XX}^{\mathrm{e}}$ siècle, et A. Elzinga a souligné le hiatus existant entre leur idéal internationaliste et la dette de leurs pratiques de recherche vis-à-vis des intérêts nationaux qui ont produit des accords comme le Traité de l'Antarctique (1993, p. 78-79, p. 83 ; voir infra «De l'instrumentalisation géopolitique des sciences»). Mais on peut justement comprendre la pratique du terrain au principe de l'ICS comme un moyen de s'acquitter de cette dette, en tant que démonstration de l'efficacité d'une coopération scientifique internationale aux bénéfices globaux. Dans la culture du terrain de l'ICS, les bases polaires figurent ainsi une espèce de poste-frontière, d'avant-poste de la civilisation - où la coopération internationale est à la fois nécessaire et dépassée, dans un humanisme scientifique porté sur le devenir de la planète.

\section{L'organisation des campagnes internationales : mutualisation et coordination}

Le travail sur le terrain est intense parce que les séjours sur la glace sont relativement courts : en Antarctique, d'octobre à février, lors de l'été austral. En outre, le coût des campagnes de forage est très élevé. Ces contraintes donnent une grande importance à leur préparation. La coopération internationale déborde donc largement

13. Liens d'autant plus remarquables que la science soviétique demeurait à l'époque très isolée, avec par exemple seulement $4 \%$ de publications avec des coauteurs étrangers dans la première partie des années 1980 (Crawford et al., 1993, p. 4). 
le terrain, et vise à définir à la fois les objectifs de la recherche et le partage des tâches scientifiques et logistiques. On peut ainsi avoir des instruments de forage dont « la France fait la mécanique, l'Italie l'électronique, avec la Suisse qui s'occupe des treuils » (t-MI). Cette division du travail marque aussi la circulation des personnes : «On peut pas dissocier l'existence des [IC] de la logistique polaire [...] Il faut des moyens logistiques lourds, il faut des bateaux, il faut des avions, il faut des véhicules à chenilles, il faut être capable de maintenir une équipe de 5 jusqu'à 40 personnes vivantes et en bonne santé dans un endroit qui n'est pas du tout fait pour l'homme [...] Et c'est l'une des raisons qui fait que cette science est très internationale. » (c-BS). Une scientifique d'un autre laboratoire français en fournit une illustration éloquente : «Cette année, je suis partie avec les Allemands [...]. Notre [matériel] est venu par icebreaker de l'Afrique du Sud à la base allemande. Nous, on a pris l'avion des Russes. On est arrivés à la base russe et, après ça, on a pris des petits avions qui sont gérés par les Canadiens pour nous emmener sur notre base allemande [...] c'est comme ça, on mélange la logistique pour que tout le monde y arrive. » (c-EZ).

Les liens tissés sur le terrain facilitent les phases de préparation, soulignent les chercheurs. Les premières décennies de l'histoire de l'ICS sont ainsi scandées par les arrangements entre les quelques «grands patrons » les plus actifs à l'époque («Les relations de pays à pays se faisaient à travers quelques individus. », c-VB), dans un cadre qui pouvait être très informel. L'un des participants se souvient «qu'on y sent[ait] guère l'esprit des drapeaux nationaux » (Lorius et Carpentier, 2010, p. 39), et la coordination demeure, dans un premier temps, plutôt informelle. Sans parler des « saunas de travail » qui les ont réunis à Saint-Pétersbourg, on peut évoquer la « réunion au sommet » de 1994 entre Français et Russes « dans la petite auberge de "Papy Garnier" au col de Porte », avec deux représentants du programme de glaciologie antarctique à la National Science Fondation (NFS) étatsunienne, et où « en une nuit, un nouveau projet de coopération est lancé » (Petit, 2013, p. 151, p. 181-182).

S'ils ont pu compter sur le soutien d'institutions comme le Scientific Committee on Antarctic Research ou l'Organisation météorologique mondiale (Turchetti et al., 2008) pour ces réunions destinées à fixer les objectifs scientifiques et discuter des moyens à leur disposition pour les prochains forages, les chercheurs se devaient néanmoins ensuite d'obtenir les financements nécessaires auprès de leurs tutelles. Les différences nationales se font alors davantage sentir, en fonction du crédit attribué à l'ICS dans chaque pays. La présence importante des chercheurs soviétiques dans l'historique de cette coordination ne doit pas cacher, par exemple, que leur employeur (l'Institut des mines de Leningrad) était bien plus intéressé par les techniques de forage et les exploits logistiques que par la production de données paléoclimatiques. Et les relations d'amitié nouées dans les espaces confinés des premiers camps polaires n'empêchaient pas leurs collègues français de bien saisir les bénéfices à en tirer. «La science des uns contre l'expérience et la logistique des autres, c'est en quelque sorte la base du marché conclu entre la France et l'URSS » dès les années 1960 (Petit, 2013, p. 81). L'un des opérateurs de ces rapprochements tire aujourd'hui « une grande fierté » (c-VB) d'avoir fait travailler ensemble Américains et Soviétiques pendant la Guerre froide, et cette coopération constitue assurément un moment fort de l'histoire de l'ICS. Il serait toutefois erroné de n'y voir qu'une conséquence des liens tissés sur le terrain. Alors que les États-Unis viennent d'afficher leur volonté de reprendre le leadership en matière de forage, C. Lorius constate ainsi, à l'époque, que «si l'on veut bénéficier du soutien de la NSF, qui est de loin plus rapide et plus efficace que la logistique soviétique, il va falloir lâcher quelque chose, donc collaborer. Mais tant qu'à faire, choisissons nos partenaires, de manière à ce que nos domaines de recherche 
ne se chevauchent pas trop » (cité dans Petit, 2013, p. 150). Le «mariage de raison entre la France et les États-Unis » de la fin des années 1980 résulte de ce constat - qui ouvre aux seconds la porte de la base soviétique de Vostok et donc à ses IC. L'un des acteurs de cette première coopération tripartite évoque «la dot de Vostok » apportée par les Français (ibid., p. 157), non sans appréhension quant à ses conséquences relationnelles sur le terrain. Mais, finalement, « au Pôle du froid, les tensions géopolitiques paraissent bien lointaines », se souvient J.-R. Petit, avant de détailler le menu d'une cohabitation joyeuse entre chercheurs des trois nations (ibid., p. 159).

La participation des États-Unis à des campagnes de forage avec les Européens et les Soviétiques trahit, en outre, la sensibilité des coopérations internationales vis-à-vis des politiques et priorités de recherche nationales dans un domaine à la logistique très couteuse, comme l'ICS. Devant la difficulté à convaincre la NSF de financer leurs activités, les scientifiques étatsuniens chercheront, en 1987, à s'associer avec les Danois, qui organiseront une réunion restée fameuse à Boston, à la suite de laquelle deux forages sont mis en œuvre, avec un partage des frais logistiques. Les deux « commenceront la même année et les résultats seront exploités en étroite collaboration » (Jouzel et al., 2008, p. 107-110). À l'inverse, le regain de crédit dont a bénéficié récemment l'ICS auprès des institutions de recherche aux États-Unis a permis à leurs scientifiques de retrouver le premier plan (Jouzel, 2013, p. 2530) et les a éloignés du reste de la communauté internationale. "Les américains, on n'a pas de projets avec eux [...] parce qu'ils ont des budgets qui sont bien supérieurs à ceux qu'on peut avoir, et ils arrivent à faire leur business de leur côté » (t-MI) ; "ayant tous les moyens logistiques disponibles, ils n'ont pas besoin forcément de créer une collaboration pour monter un projet [...] c'est ce qui peut donner l'impression qu'ils sont un peu enfermés sur eux-mêmes » (c-XB).

La coordination demeure toutefois très importante entre nations européennes, et avec les récents nouveaux venus dans le domaine de l'ICS (Jouzel et al., 2008, p. 118), et ce d'autant plus qu'il devient plus difficile d'ajouter une plus-value importante au savoir paléoclimatique, et donc de justifier a priori auprès des agences les coûts toujours aussi importants de nouveaux forages profonds ${ }^{14}$. L'un des objectifs fixés «pour les dix à vingt années à venir » par l'International Partnerships in Ice Core Sciences (IPICS), qui réunit des chercheurs de 23 nations, est ainsi «d'accéder en Antarctique à de la glace formée il y a 1,5 million d'années » (Raynaud et Chappellaz, 2008, p. 133). Mais il est très difficile de savoir où trouver cette glace, et donc impossible de solliciter dès aujourd'hui des fonds pour ce forage. «Le prix de la logistique [sur ce projet] est tel qu'il ne faut pas qu'on se plante... Enfin, si on se plante, tant pis pour nous, parce qu'on n'aura pas une deuxième chance. » (C-EZ).

L'importante coordination internationale mise en place autour de ces projets de nouveaux forages consiste donc, d'une part, à démontrer leur faisabilité technique et à concevoir de nouveaux outils permettant de connaître l'âge de la glace à plus de trois kilomètres sous la surface avec des moyens relativement légers et mobiles ${ }^{15}$, et, d'autre part, à effectuer un travail de lobbying auprès de la Commission européenne, de manière à favoriser la compatibilité des projets de la communauté ICS avec ses priorités de

14. Dans les années 1980, « la coordination était extrêmement simple. Des circuits très courts. Mais nous faisions de la science très rudimentaire. Maintenant nous faisons de la science sophistiquée, le matériau est très cher » (c-OT).

15. C'est notamment l'ambition des projets couplés «Ice and Laser » (ERC) et «Subglacior » (ANR), portés par les Français Jérôme Chappellaz et Olivier Alemany. Les scientifiques suisses, anglais et étatsuniens développent des instruments similaires (voir Witze, 2015). 
financement (celles de H2020 par exemple), tout en s'assurant du soutien des institutions de la recherche polaire (e.g. l'European Polar Board). Les entretiens avec les chercheurs confirment que cette composante de la coopération internationale (dont un modèle pourrait être donné par la communauté des astrophysiciens, c-NM) prend aujourd'hui davantage de place que lors des dernières décennies du XXe siècle, durant lesquelles le crédit institutionnel de l'ICS a cru de manière optimale, après les spectaculaires résultats des années 1980 et 1990. La création de l'IPICS, en 2004, constitue un marqueur important de cette nouvelle phase de l'histoire de la spécialité, révélateur en tout cas des nouvelles modalités et contraintes de la coopération internationale.

Les courriels échangés sur la mailing-liste dédiée au défi «oldest ice » de l'IPICS montrent, enfin, que cette arène de coordination est aussi un espace de différenciation des contributions nationales : les discussions visent notamment à définir le prix du «ticket d'entrée » des différentes nations à ce projet - autrement dit le niveau de financement que les participants devront obtenir auprès de leurs agences nationales pour s'assurer un accès privilégié aux futurs $I C$, d'un âge record.

\section{Le dispositif transnational de mobilisation et de partage des archives climatiques}

Une fois extraite du sous-sol de l'Antarctique et du Groenland, une partie des IC est stockée sur place, dans le «meilleur frigo du monde » (C-BS). D'autres sont acheminés à travers la planète, vers des laboratoires où ils vont être analysés, ou bien vers des centres de stockage associés, où ils vont demeurer à la disposition des chercheurs. Ce réseau de mobilisation des $I C$, associant continents et pays, constitue une autre manifestation du caractère transnational de l'ICS, et une composante essentielle de la «vast machine » (Edwards, 2010) assurant la globalisation du savoir climatique - plus précisément de la «sharp machine » plus pointue dessinée par l'infrastructure matérielle et institutionnelle de l'ICS.

\section{L'infrastructure de la globalisation : la « chaîne du froid » de l'ics}

Le développement de l'ICS peut être décrit, à un autre niveau, à travers la mise en place d'un réseau de partage des $I C$, une «chaîne du froid » qui constitue en quelque sorte le système nerveux de la communauté ${ }^{16}$. L'organisation de ce réseau se fait dans le prolongement de celle des campagnes de forage, déterminant la distribution des objets et la prise en charge de leur acheminement, par air, mer et route - et renforce encore le maillage international de l'ICS.

16. Dans une perspective « marquée par les sciences de terrain », Simon Schaffer a montré l'intérêt que ce type de dispositif pouvait présenter (en tant qu' «agencement matériel et normatif ») pour la compréhension de la dynamique de «globalisation » des sciences (Van Damme, 2014). D'autres historiens souhaitant se défaire d'une focale trop portée sur les innovations conceptuelles ont également analysé comment une communauté scientifique pouvait émerger en se saisissant d'un nouvel objet et en organisant sa circulation entre diverses équipes de recherche, i.e. en transformant un objet naturel en « instrument de production scientifique» (voir le travail de R. E. Kohler, 1994, p. 168, sur la mouche drosophile : le dispositif d'échange y « incarne les principes moraux et sociaux » définissant la communauté des «drosophilistes », en matérialisant « des pratiques standards et un mode de vie expérimentale propres »). 
Le compte rendu d'une campagne de forage publié dans Nature illustre «l'extraordinaire logistique » qui permet d'acheminer les $I C$ vers les «centres de calcul» (Latour, 1989) que sont les laboratoires, et où ils seront détruits lors de «manips » visant à en analyser la composition : cette logistique «impliquait des mécaniciens, des foreurs et scientifiques de cinq nations, ainsi que le stockage et le transport de plus de $2500 \mathrm{~m}^{3}$ de glace. La glace a d'abord été transportée en avion [du site de forage] à la base côtière italienne [en Antarctique], puis ramenée par bateau dans les laboratoires européens - à des températures maintenues sous les $-25^{\circ} \mathrm{C}$ afin de préserver le signal climatique dans la glace. Les IC ont été découpés et traités [dans un centre de recherche allemand] » (Frezzotti, 2011). Il n'est donc pas surprenant que les hommages à la logistique soient fréquents dans les récits des chercheurs. Richard B. Alley ([2000] 2014) écrit, par exemple, que les moyens aéroportés de la $109^{\mathrm{e}}$ division de la garde nationale étatsunienne « ont révolutionné la recherche polaire ». L'un de ses collègues français est aussi admiratif des Hercules C130 étatsuniens que des Kharkovtchanka soviétiques - des camions à chenilles de plus de 30 tonnes (Petit, 2013).

On voit aussi, encore une fois, que la propriété de ces moyens de transport adaptés (d'origine militaire, le plus souvent), comme celles de bases permanentes au Groenland et plus encore en Antarctique, introduit une certaine asymétrie dans les négociations entre scientifiques, qui se rapporte à des différences nationales. La géographie du domaine, avec ses flux migratoires et ses centres de décision et de traitement, est de ce point de vue assez éloignée du «tissu sans couture » parfois superficiellement associé à la globalisation de et par les sciences, mais aussi de la Cité scientifique idéale qui se matérialise aux yeux des acteurs sur le terrain. Ce constat est encore renforcé lorsqu'on considère le processus d'attribution de la glace stockée à proximité des laboratoires, les modes d'accès aux objets scientifiques.

\section{Le partage des IC : coopérations et concurrences dans le réseau}

« Si tu as la glace, on viendra te demander, tu es un interlocuteur indispensable et tu peux discuter les termes de la collaboration. », disait C. Lorius après s'être ménagé un accès à de précieux IC forés par ses amis soviétiques (Petit, 2013, p. 151). "Pour garder un petit peu la maîtrise des choses ou être toujours dans le coup, pour ne pas être mis à l'écart, il faut avoir les [IC]. », confirme l'un de ses collègues (c-OT). L'accès aux échantillons est aujourd'hui plus formalisé que lors des premières décennies de $l^{\prime} I C S^{17}$, et la question de l'accès aux objets scientifiques en grande partie décidée avant même les forages, lors de la phase de préparation des campagnes, en fonction des projets, savoir-faire, et moyens des uns et des autres. Mais si la moitié des échantillons fait ainsi « le tour du monde des paillasses » (Jouzel et Debroise, 2007, p. 32), l'autre moitié est conservée comme archives, soit sur le terrain, soit sur les étagères d'entrepôts de stockage où ils peuvent rester plusieurs années avant d'être analysés.

Les campagnes de forage visent donc plus largement à nourrir un stock d'archives climatiques, a priori disponible pour l'ensemble de la communauté internationale. La participation aux grandes campagnes sur le terrain assure ainsi non seulement un

17. L'évolution de ces formalités témoigne de l'inventivité des scientifiques en matière de régulation des réseaux de recherche. Maurice Cassier (1998) a dessiné un programme de recherche sur le sujet, à partir de l'analyse d'un dispositif de gestion de la concurrence et de la coopération, dans un réseau mêlant laboratoires de biotechnologie publics et privés. 
accès direct aux $I C$, mais aussi à la gestion de ce stock, via les conseils des consortiums auxquels les chercheurs doivent formuler leurs demandes pour disposer des échantillons qui les intéressent. "L'argent et la science sont intimement mêlés, quelque part... [...] parce que pour avoir accès à un $[I C]$, il faut avoir payé au début, pour la logistique. Et donc après c'est pas n'importe qui qui pourra avoir accès à la glace et publier n'importe quoi. Donc c'est pour ça, c'est une sorte de contrôle les uns des autres, qu'on dépasse pas nos frontières et nos droits. » (c-KS). Cette composante de l'infrastructure permet à la communauté de gérer l'exploitation scientifique des $I C$, à l'intérieur et éventuellement au-delà du périmètre du consortium propriétaire, et ainsi de conjurer les limites de son savoir-faire sur les plans synchronique - à la même époque, d'autres équipes peuvent produire des analyses alternatives - et diachronique - la constante évolution des instruments permet d'envisager que les IC fassent un jour l'objet de nouvelles analyses, imprévues au départ ${ }^{18}$.

« Dans l'échantillon, il y a de l'or scientifique [...] c'est un peu la Pierre de Lune. » (c-LM) ; "Quand vous avez de la glace [issue d'un forage profond], c'est un peu comme un morceau de lune ou un morceau de comète. » (c-OT)... Cette rareté, conjuguée à la fragilité de la glace, implique un « stewardship $»^{19}$ collectif, distribué à tous les niveaux de la communauté. Cette responsabilité débute avec l'autocensure des chercheurs, qui constitue une forme de régulation passive du stock, notamment lorsque la glace convoitée est rare (parce que forée en profondeur ${ }^{20}$ et/ou dans un endroit peu accessible). «On s'autocensure... car [le risque c'est que] quelques années plus tard, on a quelqu'un qui arrive avec une super machine ou une super idée et on aura gaspillé cette glace pour rien. » (c-PL). Une autre chercheuse indique, dans un courriel de demande de glace supplémentaire au comité scientifique d'un projet, qu'elle ne préfère demander, dans un premier temps, que peu d'échantillons de glace profonde, « pour ne pas la gâcher » en risquant de la stocker dans un endroit pas complètement fiable pendant la mise en place de ses manips. «Il y a ce côté précieux. La glace, il y en n'a pas beaucoup, on n'ira pas reforer là où on a déjà foré parce que ça coûte des millions, donc on ira toujours dans un nouvel endroit. Et du coup, si on se plante, il y a un trou dans la courbe. Donc ça, ça met la pression quand même. » (c-EZ).

Les échanges de courriels entre chercheurs d'un projet international en cours révèlent une coordination en poupées russes : de nombreuses discussions ont par exemple lieu entre les membres du groupe responsable des analyses du gaz piégé dans les $I C$, afin de rassembler les nouvelles demandes et éliminer les conflits, avant de présenter une soumission groupée de glace supplémentaire au comité scientifique du consortium. Dans ces échanges, le responsable de la formalisation de cette demande est

18. Cette marge de manœuvre peut être exploitée dès l'extraction des IC sur le terrain, pour ajouter des manips à la liste prévue au départ du projet. Un échange de courriels initié par un chercheur depuis l'Antarctique aboutit ainsi à l'envoi d'une demande au steering committee de ce projet, dans laquelle il présente l'intérêt scientifique des manips qu'il souhaite effectuer sur place, mais aussi les conditions idéales dans lesquelles il se trouve pour les réaliser (températures locales, instruments installés, etc.), ainsi que sa longue expérience en la matière.

19. J'emprunte le terme au lexique muséal. Le stock de glace d'un laboratoire est confié à un « curateur » (terme indigène, également usuel dans les musées) local, qui a notamment la responsabilité des fichiers de tableurs répertoriant les échantillons, leurs caractéristiques (longueur, provenance, etc.), lieux de stockage, et éventuelles attributions à des équipes. J'ai pu constater que ces fichiers font l'objet d'un grand soin et de nombreux échanges (il est notamment crucial de savoir combien de centimètres il reste de chaque échantillon).

20. Les $I C$ les plus longs, i.e. ceux permettant de remonter jusqu'à la dernière période interglaciaire, sont bien sûr les plus rares : sept forés au Groenland et une douzaine en Antarctique (Jouzel, 2013, p. 2525). 
amené à prévenir ses collègues : « S'il ne reste que 25 ou 30 centimètres [de tel échantillon], nous aurons à discuter... » Une autre requête imprévue l'oblige à rappeler la « politique commune » consistant à conserver des échantillons de toutes les sections de l'IC (« même si je trouve le contenu de votre projet passionnant, il me pose aussi un problème dans la mesure où il implique l'utilisation de toute la glace restant dans ces sections ») et à leur demander s'ils pourraient maintenir leurs manips avec seulement deux tiers des sections demandées. Ces échanges concernent aussi le moment et les moyens du transfert entre le terrain (où les conditions de conservation des $I C$ sont parfaites et peu coûteuses) et les laboratoires, et ce d'autant plus que certaines manips nécessitent le maintien des $I C$ à de très basses températures (jusqu'à $-50^{\circ} \mathrm{C}$ ) et donc des moyens de transport et de stockage spécifiques.

On voit que ce réseau de mobilisation des $I C$ assure le prolongement de la coordination internationale des équipes de chercheurs bien au-delà des campagnes de forage sur le terrain, en favorisant une importante circulation d'informations au sein de la communauté. Accéder à de la glace supplémentaire nécessite en effet de développer une (brève) argumentation scientifique à destination des pairs, afin de les convaincre de l'intérêt de la destruction d'une partie des archives glaciaires déjà conditionnées : les justifications avancées lors de ces demandes donnent dès lors une visibilité a minima aux projets des uns et des autres, en aval du terrain cette fois. Dans certains cas, des coopérations peuvent émerger lorsque les manips envisagées par des collègues sont proches. Enfin, si la glace circule souvent grâce aux services de transporteurs spécialisés, il est fréquent que les chercheurs se déplacent eux-mêmes pour la découper (un autre rituel, comme j'ai pu le constater), et les rencontres sur place avec des collègues constituent des occasions supplémentaires de discuter des manips prévues, hors des circuits académiques plus classiques dessinés par les colloques et publications ${ }^{21}$.

La gestion du flux des IC apparaît de ce point de vue comme un instrument collectif de coordination internationale adapté aux spécificités des objets, voire comme une infrastructure permettant de corriger les éventuels travers d'un accès inégal au terrain, même si la rareté de la glace peut entraîner certaines tensions dans un contexte de compétition scientifique classique ${ }^{22}$ (notamment lorsqu'une équipe dont les tutelles nationales ne contribuent pas aux campagnes de forage oublie «la coutume » [t-RR] et n'hésite pas à multiplier les demandes sur le « second marché » de l'économie des IC).

\section{L'ICS dans le contexte des relations internationales : espaces naturels et projections politiques}

La dynamique internationale de l'ICS renvoie à un autre niveau encore, qui dépasse cette fois l'organisation de sa communauté scientifique. Si les particularités naturelles de leurs terrains de prédilection et des objets qu'ils manipulent marquent inévitablement la culture professionnelle des paléoclimatologues, l'histoire géopolitique du Groenland et de l'Antarctique constitue également un facteur qu'il est impossible de négliger lorsqu'on veut saisir les formes et le sens du «fait international » dans ce domaine.

21. De même, «l'état de l'art » de la physique des hautes énergies se transmet oralement, autour des instruments (Traweek, 1992, p. 126).

22. «On est toujours entre la collaboration et la compétition [...] et la frontière est ténue. » (c-DW); «La compétition est toujours là, et on la sent. » (c-PL). 
Les liens privilégiés du Danemark avec le Groenland expliquent par exemple que les chercheurs danois soient très présents dans l'histoire arctique de l'ICS, suivant une logique qui ne peut être seulement celle d'un goût invariant de ses gouvernants pour la paléoclimatologie. De même, l'installation d'une base étatsunienne au Groenland, en 1958, où auront lieu les premiers forages glaciaires, dépend moins des ressources scientifiques locales que de sa localisation sur la route la plus directe entre les ÉtatsUnis et l'URSS. Mais cette influence géopolitique est peut-être plus manifeste encore pour ce qui concerne les activités scientifiques qui se sont déployées sur le plus lointain continent Antarctique, sur lequel je vais concentrer cette dernière partie.

\section{De l'instrumentalisation géopolitique des sciences}

L'histoire des sciences en Antarctique est marquée par deux événements rapprochés : l'organisation de l'Année géophysique internationale (1957-1958), qui a permis d'organiser de vastes campagnes de mesures sur le terrain et, dans son prolongement, la signature du Traité de l'Antarctique en 1959 (ratifié en 1961 par douze pays), qui a fait de ce continent un espace exclusivement réservé aux activités scientifiques (Pyne, [1987] 2004 ; Fogg, 1992 ; Elzinga, 1993 ; Launius et al., 2010 ; Walton, 2013). Depuis, cette période est souvent présentée comme celle de l'expérience réussie d'un « dégel » géopolitique sur un territoire disputé de la Guerre froide ${ }^{23}$, par la magie de la coopération scientifique internationale.

L'exploitation d'archives récemment déclassées a permis de préciser les fondements géostratégiques du « régime de coopération » dont les «polaires » ont bénéficié à l'époque, liés à l'intérêt militaire et commercial du contrôle des océans environnant l'Antarctique, et à la «menace spécifique représentée par la présence de bases soviétiques » sur place. C'est uniquement parce que ces motivations n'étaient connues que de quelques diplomates que ce régime a pu apparaître comme l'expression de l' « internationalisme scientifique» (Turchetti et al., 2008, p. 351-352, p. 368) ${ }^{24}$. «En Antarctique, la science [...] a représenté une façon de maintenir une présence sur le continent [...] et de collecter de l'intelligence géostratégique. » (Naylor et al., 2008.) Ainsi, la définition de l'Antarctique comme terrain réservé aux activités scientifiques ne signifie pas que les rivalités internationales y ont été subitement effacées, mais plutôt qu'elles ont été à la fois déplacées sur le terrain scientifique - et finalement atténuées par les nécessités de la coopération qui s'imposent sur ce terrain hostile et difficile d'accès (voir infra «La Cité scientifique sur la glace : solidarité et science "extrême" ») ${ }^{25}$. Le Traité de l'Antarctique est plutôt considéré aujourd'hui comme manifestant une opportuniste «exploitation politique de la bonne volonté scientifique », permettant non pas de mettre un terme aux «visées impérialistes » mais de les «geler», selon le calembour officiel de son article 4 (Howkins, 2010, p. 246, p. 260-261).

23. Voir, par exemple, les préface et postface de Margaret Thatcher et Michel Rocard («La force de l'amitié a sauvé l'Antarctique ») aux ouvrages de Gordon E. Fogg (1992) et de C. Lorius et Laurent Carpentier (2010).

24. Voir, par exemple, l'ouvrage de C. Richard Lewis et Philip M. Smith, 1973, Frozen Future. A Prophetic Report from Antarctica, qui présente ce régime comme « une nouvelle expérience de civilisation » fondée sur des relations pacifiques.

25. De la même façon, le partage des données satellitaires et le concept du World Weather Watch sont «directement issus» de la politique de la Guerre froide, dans le sens où ils ont été conçus comme « contrepoids aux tensions militaires et idéologiques » (Edwards, 2010, p. 14). 
Ces accords conditionnent la participation aux discussions sur le statut de l'Antarctique à l'envoi de chercheurs sur place, et ceux de l'ICS peuvent avoir le sentiment légitime de figurer parmi les enfants du Traité (Lorius et Carpentier, 2010, p. 14). Si, à l'époque, « l'idée que les calottes polaires contiennent des archives privilégiées est encore peu présente dans les programmes » (Jouzel et al., 2008, p. 97), elle est tout de même déjà exprimée par les scientifiques (Lewis, 1965, p. 146 sq.), et les crédits alloués à cette occasion leur donnent la possibilité de mener une exploration in situ, en s'appuyant sur des travaux réalisés à partir d'idées exprimées en 1954 par le Danois Willi Dansgaard sur la composition isotopique de la glace (Dansgaard, 2005). Ces crédits « permettront notamment de développer des carottiers » (Jouzel et Debroise, 2007, p. 26) et, dans un livre consacré aux sciences en Antarctique et écrit peu de temps après, les tubes ramenés en 1958 par l'uss Glacier et contenant les sections du premier IC profond étatsuniens sont décrits comme « les données brutes de l'une des plus ambitieuses expériences de l'AGI »(Lewis, 1965, p. 146).

Le soutien politique apporté alors aux chercheurs qui vont définir les bases cognitives et matérielles de l'ICS s'appuie en outre sur la dimension symbolique attachée, dans de nombreux pays, à l'exploration d'un territoire encore peu connu à l'époque. Dans cette optique, «les prouesses [des scientifiques] pouvaient symboliser la supériorité d'une idéologie politique sur une autre» (Naylor et al., 2008), être perçues comme l'expression d'un «soft power» (Nye, 2004) à une époque associant étroitement puissance nationale et avancées scientifiques et technologiques (Oreskes et Krige, 2014). Les expéditions scientifiques ont ainsi pu être perçues comme des "prouesses nationales, pour essayer de montrer qu'un système était mieux que l'autre », comme une « démonstration de la capacité de pouvoir aller n'importe où, où on veut, sur la planète » $(\mathrm{c}-\mathrm{XB})$. Ainsi, la logique d'implantation des bases à partir desquelles les chercheurs ont travaillé trahit la perméabilité des sciences polaires vis-à-vis de ces logiques de conquête : «Les États-Unis, par exemple, ont revendiqué le pôle Sud symbolique comme le site de l'une de leur station pendant l'AGI, ne laissant aux Soviétiques que les moins prestigieux points de relative inaccessibilité et pôle géomagnétique. » (Naylor et al., 2008, p. 145). D'un autre côté, si la construction d'une base dans les conditions extrêmes du pôle Sud fut « un exemple classique de vanité technologique et scientifique » étatsunien, cet effort a sans doute été dépassé par l'installation des Soviétiques sur le site le plus éloigné des côtes antarctiques, le point d'inaccessibilité (Howkins, 2010, p. 255).

La spectaculaire logistique des expéditions polaires se prête aussi à ce type de valorisation, inscrivant les avancées des scientifiques dans le lignage des exploits des premiers explorateurs (qui, pour certains, ont accédé au statut de héros nationaux). Le développement de l'ICS, en particulier, s'est appuyé sur la mise en œuvre de forages (dans des zones reculées, sur des sommets affichant des records de froid et de vent, etc.) pouvant être assimilés à des exploits techniques et humains. À la fin des années 1960, l'inlandsis de l'Antarctique voit ainsi «se jouer une forme de défi à distance entre les foreurs du Cold Regions Research and Engineering Laboratory de l'armée étatsunienne et ceux de l'Institut des mines de Leningrad » (Petit, 2013, p. 86). «Les Soviétiques, ils faisaient tout. Par principe. Ils étaient donc très forts en logistique. [Ils ont fait des millions de kilomètres] simplement pour mettre le drapeau. Donc [...] ils sont allés au point d'inaccessibilité, ils [y] ont mis une statue de Lénine [...]. On est en pleine Guerre froide, les Américains ont atteint 2138 mètres à Byrd. Le programme des Soviétiques à Vostok, c'est de forer dans la glace. Pourquoi ? Pour atteindre 2200 mètres. » (c-OT). À l'inverse de l'image de Cité idéale associée aux bases polaires (voir infra «La Cité scientifique sur la glace: solidarité et science 
"extrême" »), qui propose un dépassement des rivalités du point de vue des scientifiques, la valorisation de ces exploits "nationaux » a plutôt contribué à effacer les traces des coopérations sur le terrain ${ }^{26}$.

L'intérêt national à soutenir les sciences polaires dépasse toutefois le plan symbolique du « rayonnement national» (au sens de Hecht, [1998] 2004). L'importance des moyens techniques nécessaires aux scientifiques pour se rendre et travailler en Antarctique permet en effet également aux militaires de demeurer sur place, au second plan, en soutien des activités scientifiques. Ce soutien constitue donc aussi un moyen de poursuivre, comme en sourdine, le déploiement de ressources stratégiques. De nombreux travaux ont montré comment les recherches effectuées sur place bénéficient souvent du patronage ambivalent des militaires (Grevsmühl, 2014) : contrainte et ressource, il permet aux scientifiques de travailler, durant la Guerre froide, en « semiautonomie », sur des «technologiques expérimentales » à la frontière «entre recherches fondamentales et appliquées, militaire et civiles» (Krige, 2014a, p. 431-433). Les récits des chercheurs de la première génération ne font pas mention d'un dialogue portant sur autre chose que l'indispensable mise à disposition de leurs véhicules. Si cette dimension presque exclusivement logistique du lien entre les militaires et les scientifiques de l'ICS les a forcément moins contraints que leurs collègues financés pour travailler sur la haute atmosphère (pour les fusées) ou la salinité de l'eau (pour les sous-marins $)^{27}$, par exemple, elle n'était toutefois pas sans importance pour les premiers. La logistique polaire a constitué, à l'époque, un « levier important » d'une administration étatsunienne soucieuse de " jouer un rôle moteur dans l'organisation des affaires antarctiques » (Turchetti et al., 2008, p. 353 ; voir aussi Belanger, 2006). Et il paraît évident que les campagnes des paléoclimatologues offraient d'instructifs tests in situ pour les avions Hercules C130 de l'us Air Force et leurs nouveaux instruments de navigation ${ }^{28}$, ou pour les impressionnants tracteurs polaires soviétiques. Un participant aux premières expéditions tripartites (France-EU-URSS) raconte en outre que «le commissaire politique de la base [soviétique] venait aussi voir les avions américains... » (c-VB).

Saisir la dynamique d'une science «de terrain » comme l'ICS entraîne ainsi non seulement à considérer les particularités naturelles de l'environnement mais aussi son histoire politique, qui expose les chercheurs à des ressources et contraintes déterminantes. Dans ce cas, on comprend aussi l'intérêt que des pays qui avaient auparavant un pied stratégiquement posé en Antarctique, comme la France, ont trouvé à soutenir l'insertion de leurs chercheurs dans les programmes internationaux initiés avec l'AGI - sans pour autant que leurs autorités s'intéressent de près au contenu des recherches (Elzinga, 1993, p. 96). La fibre internationale de l'ICS apparaît ici sous un jour plus contrasté, entremêlant les fils des coopérations scientifique et politique sur un canevas dessiné par des rivalités stratégiques ${ }^{29}$.

26. C'est aussi ce que montre John Krige (2014b, p. 227-228) à propos de la célébration, par la France, du lancement de sa fusée Véronique, en 1959.

27. Naomi Oreskes (2014a) souligne combien il a été difficile, pour des domaines que les militaires ont beaucoup soutenu pendant la Guerre froide, de changer de «contexte de motivation ». Sur la question de l'autonomie des scientifiques dans ce type de contexte, voir l'analyse de Simone Turchetti et Peder Roberts (2014a, p. 8-12).

28. «Sans le vouloir, les militaires ont fait énormément progresser notre domaine parce qu'ils ont toujours voulu avoir de l'électronique qui résistait à $-50^{\circ}$. Pour d'autres raisons bien sûr. Et donc ça a servi énormément dans toute la technologie qu'on utilisait en Antarctique. » (c-GL).

29. De même, dans le domaine des technologies et sciences spatiales des années 1960 et 1970, « les motifs de la collaboration internationale »sont étroitement dépendants des politiques étrangères 


\section{L'Ics et le " régime climatique »}

De manière imprévisible au moment de la signature du Traité de l'Antarctique, le développement des sciences du climat va faire évoluer ce cadre géopolitique. L'exemple le plus documenté est celui des recherches menées sur la stratosphère depuis le pôle Sud, qui ont permis de révéler l'existence d'un «trou » dans la couche d'ozone en 1985. Les travaux réalisés à la même époque par les paléoclimatologues de l'ICS, sur les cycles climatiques et l'impact des gaz à effets de serre, ont aussi grandement contribué à changer le statut des territoires polaires. S'interrogeant sur «l'invention de l'environnement global», Sebastian Vincent Grevsmühl (2014, p. 15) a ainsi souligné « le rôle historique de la région antarctique dans le changement profond de nos conceptions spatiales globales, notamment dans les discours portant sur les limites de la Terre et la façon dont il faut les appréhender ». Inhabité, l'Antarctique était encore largement inconnu avant l'AGI (Howkins, 2010, p. 245, p. 256), et l'on peut dès lors dire qu'il est devenu ensuite rapidement « partie intégrante de l'économie mondiale de la connaissance » (Pyne, [1987] 2004, p. 325), « qu'il a été construit par la science » (Elzinga, 1993, p. 77) - une science de plus en plus orientée vers la problématique du changement climatique (Howkins, 2011) ${ }^{30}$.

Contrairement à celui de l'AGI de 1957-1958 imprégné par la Guerre froide, « le contexte politique» de "l'Année polaire » internationale de 2007 a été marqué par la thématique du «changement climatique », constatait à son terme un éditorialiste de Nature $^{31}$. L'observation peut aujourd'hui paraître très banale. Elle cache en fait un retournement de grande ampleur, qui a touché l'investissement des scientifiques sur les terrains polaires, les conditions politiques de leur soutien et le sens de la coopération internationale entre paléoclimatologues.

Lors de ces dernières décennies, la question de la pollution environnementale globale s'est affirmée comme une problématique majeure dans l'espace public (voir, par exemple, McNeill, 2010). Cette question «a émergé publiquement à la fois comme une préoccupation de l'opinion, un enjeu majeur de la politique des États et un domaine scientifique » (Ingold, 2011, p. 11), et, pour de nombreux observateurs, elle a sensiblement redéfini le « contrat science-société », renouvelé les relations entre « savoir et pouvoir» (Pestre, 2010 ; Ravetz, 2006). Cette évolution s'est exprimée très visiblement avec le développement des sciences du climat, qui ont, pendant cette période, dissipé nombre d'incertitudes pesant sur la nature du changement climatique et le rôle des gaz à effets à serre dans le réchauffement en cours (Weart, 2008 ; Oreskes, 2004). Dans la littérature STS, le caractère paradigmatique de ce développement n'est pas lié à l'accroissement des publications sur le sujet, mais aux «implications politiques majeures » de cette connaissance, qui explique qu' " une forte interaction existe aujourd'hui entre la science et les décisions politiques, les discours des groupes d'intérêt, qu'ils soient pro-industrie ou pro-environnement »(Gingras, 2010, p. 126). Cette interaction a conduit plusieurs auteurs à évoquer l'émergence d'un « régime climatique » (Aykut et Dahan, 2014) dont la généalogie est désormais bien établie, depuis la première Conférence mondiale sur

des gouvernements, qui inscrivent «les relations géopolitiques [...] dans la circulation globale du savoir » (Krige, 2014b, p. 230).

30. Pour l'Arctique, voir Doel et al. (2014) et Martin-Nielsen (2013).

31. «The Ends of the Earth. International Polar Year 2007 Can Leave an Imprint », Nature, 446, 110, 8 March 2007, p. 110. 
le climat de Genève (1979) à la signature de la convention-cadre de Rio (1992), en passant par la création, en 1988, du Groupe d'experts intergouvernemental sur l'évolution du climat (GIEC).

Il n'est pas question de détailler cette évolution ici, mais de souligner que l'émergence de ce « régime climatique » a transformé la géopolitique internationale, et donc la façon dont les États se projettent sur les territoires polaires, en même temps que les relations entre les communautés scientifiques et leurs tutelles politiques. À la suite du Traité de l'Antarctique, la science est mobilisée comme un adjuvant politique de « dégel », afin de désamorcer un conflit territorial latent (voir infra « De l'instrumentalisation géopolitique des sciences ») ; elle s'est affirmée depuis comme une source de connaissances incitant à une action internationale, la plus coopérative possible - voire une réserve de "capital symbolique politique », comme l'indique justement A. Elzinga (1993, p. 79) au sujet de l'ICS. Autrement dit, si la géopolitique internationale pèse autant sur les activités de l'ICS, elle n'a plus du tout le même sens qu'au moment des premières campagnes. Suivant la formule de Bruno Latour, « qu'un prix Nobel de la Paix vienne récompenser le GIEC [en 2007] prouve assez que c'est bien toute la géopolitique qui se trouve agitée par une question de modélisation mathématique du climat de la Terre » (2008, p. 659). Mais il faut aussi rappeler que, pendant la Guerre froide, ce sont plutôt les sciences - et en particulier celles de la Terre (Doel, 1997, 2003 ; Turchetti et Roberts, 2014b) - qui se trouvent « agitées » par des questions géopolitiques.

Il est certes discutable que ce retournement relève de la seule action des scientifiques (Bonneuil et Fressoz, 2013). Il paraît difficile, en revanche, de nier que leur contribution a été décisive, et l'ICS figure en bonne place parmi les spécialités qui ont permis de mieux comprendre la dynamique du climat terrestre (notamment en suivant les variations, dans l'atmosphère, des gaz à effet de serre comme le co2 et le méthane) et d'objectiver la sensibilité du climat terrestre vis-à-vis de certains « forçages ». De ce point de vue, l'ICS a transformé son terrain polaire et changé sa perception dans les relations internationales : le travail et les témoignages de ses chercheurs ont donné de nouvelles raisons à la protection politique des pôles et de leurs sciences, ouvrant « une porte sur un problème aujourd'hui au cœur des préoccupations de notre société » (Jouzel et al., 2008, p. 280). Alors que, comme l'indique C. Lorius, personne ne "s'inquiète de l'atmosphère du passé en 1965 » (Lorius et Carpentier, 2010, p. 38), la situation est sensiblement différente en 1987, lorsque Nature fait sa couverture avec les résultats de son équipe sur la corrélation entre hausse des températures et teneur en gaz à effet de serre, produisant des graphiques qui ont fait ensuite «le tour du monde, y compris des sphères politiques » (Jouzel et al., 2008, p. 122), et sont devenus des « icônes » du GIEC (c-GL). " [Au milieu des années 1970], les gens rigolaient ! "Eh, tu coinces la bulle dans les glaces !", bon, ce genre de plaisanteries... ou : "c'est pas sérieux ces trucs-là dans la glace”... [Aujourd'hui] on est reconnus... on est devenus une science, tout le monde en veut. Pour nous, ça a été vraiment extraordinaire comme aventure. Parce qu'on est partis de quelque chose d'anecdotique à une discipline reconnue. » (c-Ev). En France, le niveau de cette reconnaissance est frappant : malgré sa petite taille (une vingtaine de titulaires), la communauté française compte en effet deux médailles d'or CNRS (2002), une médaille Niels Bohr (2014), un prix Vetlesen (considéré comme le « Nobel des sciences de la Terre et de l'Univers », 2012), un prix Irène Joliot-Curie ( "Femme scientifique française de l'année», 2013)... Et, aujourd'hui, les acteurs médiatiques et politiques reprennent effectivement sans hésiter les formules des livres des chercheurs de l'ICS qui, un pied dans leur laboratoire, un autre sur le 
terrain $^{32}$, présentent les glaces polaires comme un observatoire des différences entre holocène et «anthropocène », des « sentinelles de notre environnement », des «fenêtres sur notre planète », un «balcon avec vue imprenable sur la Terre », des " témoins et acteurs essentiels du réchauffement climatique », etc. (Hauglustaine et al., 2008 ; Lorius et Carpentier, 2010 ; Jouzel et al., 2008).

Les historiens de l'environnement ont montré comment la fluidification (au sens littéral) du blé avait transformé à la fois Chicago et ses terres d'origine (Cronon, 1991), ou comment le déplacement des graines rapportées des colonies avait transformé la conscience « écologique » des colonisateurs (Grove, [1993] 2013). De même, le flux des $I C$ entre pôles et laboratoires a contribué à changer la nature des pôles.

Finalement, l'ICS apparaît comme un domaine dont les animateurs ont su construire, à partir du cadrage politique souple de leurs investissements initiaux sur le terrain, et en quelques décennies, une crédibilité leur permettant de s'en émanciper - et ainsi survivre à la disparition des logiques politiques de mobilisation de la Guerre froide. Cette analyse conduit à donner raison à J. Krige (2014a, p. 431) quand il avance (versus Forman, 1987) que les scientifiques sont parvenus à se défaire d'une relation de dépendance vis-à-vis des autorités politiques en mettant à profit les « formes et termes de leur intégration sociale » durant la Guerre froide, dans la mesure où les chercheurs de l'ICS ont trouvé dans ce cadre un motif justifiant leur financement au-delà ${ }^{33}$. «On a été les passagers heureux d'une géopolitique dont on avait rien à faire. Et [...] on a donné les justifications scientifiques... qui nous permettent de faire [de la science]... » résume une scientifique (c-EZ). L'émergence du souci climatique et la volonté politique de promouvoir la coopération scientifique, au niveau européen, ont été les catalyseurs de cette transition, entre les années 1980 et 1990 : les résultats produits par l'ICS à la fin des années 1980 ont été tellement forts qu' «il y a eu une confiance assez spontanée qui a été mise par l'Europe dans cette communauté scientifique »(c-BS). «La valeur de ce domaine a été très bien reconnue. », confirme un autre chercheur: "L'Europe a mis beaucoup d'argent pour nos projets dans les $4^{\mathrm{e}}$ et $5^{\mathrm{e}}$ PCRD. » (r-LM).

Cela dit, l'impact des connaissances paléoclimatiques sur les relations internationales n'est pas uniforme : il ne s'agit pas de dire qu'il embarque toutes les nations dans un même mouvement de sauvegarde de la planète. S'il leur est difficile d'ignorer ce discours scientifique magnifié par des institutions internationales comme le GIEC (qui a eu «un effet de levier important» pour la crédibilité de l'ICS, c-BS), leurs gouvernements y sont plus ou moins sensibles (Aykut et Dahan, 2014). Ce discours pourrait même, dans un avenir proche, être pris dans de nouvelles luttes géopolitiques potentiellement révolutionnaires à l'échelle des pôles, si les difficultés techniques (du forage à travers la glace), les barrières naturelles (via la fonte de la glace) ou réglementaires (notamment la fin du moratoire du Protocole de Madrid, signé en 1991) interdisant l'exploitation des ressources énergétiques en Antarctique étaient amenées à disparaître. Le paradoxe est en effet que la contribution de certains pays aux progrès des connaissances climatiques - et donc à l'image des pôles comme «sentinelles du

32. Les vertus du contact direct avec le terrain donnent au discours des scientifiques un poids qu'il ne saurait avoir s'ils demeuraient dans leurs laboratoires (voir White, 1996 ; Hevly, 1996). Les figures de l'ICS conjuguent, de ce point de vue, deux types de crédibilité (sur l'histoire entremêlée et antagoniste de ces types, voir Kohler, 2002, p. 9-10 notamment). «C'est un peu Darwin quoi. On va dans un endroit loin, on découpe de la glace, on découvre quelque chose de nouveau. » (c-LM).

33. L'une des thématiques de l'ouvrage que J. Krige conclut est justement la capacité de certains domaines (l'océanographie, la géochimie isotopique) ou institutions (e.g. la NASA) à trouver de tels motifs, entre Guerre froide et changement climatique. 
climat »- a probablement reposé, et repose encore aujourd'hui, sur cette probabilité d'une exploitation future. On voit à nouveau combien la coopération scientifique internationale sur le terrain peut s'appuyer sur des logiques très diverses.

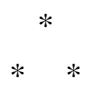

La variété des manifestations empiriques de l'internationalité de l'ICS apparaît comme un levier heuristique permettant de révéler l'influence des spécificités de l'objet scientifique sur l'organisation d'un domaine de recherche, aussi bien que la sensibilité des formes de coopération entre chercheurs vis-à-vis d'enjeux géopolitiques (liés aux régions polaires, dans leur cas).

Au-delà du constat de la diversité des facteurs de développement des spécialités scientifiques $^{34}$, l'éclatement du «fait international » constitue un point d'appui empirique pour des réflexions sur le traitement du contexte social et de l'historicité des sciences en STS. Je voudrais donc, pour conclure cet article, apporter quelques éléments de réflexion sur cette question, curieusement sous-investie en histoire des sciences (dixit Shapin, 1992 ; Galison et Stump, 1996 ; Galison, 2008 ; Oreskes, 2014b). Par rapport aux approches «macro » ou «micro » qui ont rythmé le courant alternatif dominant de la sociologie des sciences de ces dernières décennies (Gingras, 2013), la sociologie de ces objets « intermédiaires » que sont les spécialités scientifiques incite en effet plus évidemment à se rapprocher de la formule «multiscopique » (Rosental, 1996, p. 141) qui a nourri «la redéfinition de la notion de contexte» en histoire, périmant notamment l'idée " qu'il existerait un contexte unifié, homogène », au profit d'une prise en compte de leur pluralité (Revel, 1996, p. 25-26, p. 30). Plutôt que de découper l'histoire de l'ICS en " coupes temporelles régulièrement espacées pour faire l'inventaire de leurs ressemblances et de leurs différences », j'ai préféré adopter ici le modèle braudélien (ibid., p. 33-34) du « tableau en mouvement» exploitant la variété des points de vue sur l'objet : c'est l'une des vertus du « jeu des échelles » permettant d' «identifier les systèmes de contextes » dans lesquels agissent les acteurs (Lepetit, 1996, p. 79-81).

Les études transnationales incitent à « jouer plus explicitement avec les échelles » (Pestre, 2012), et la sociologie historique des sciences esquissée dans ce texte implique en effet de prendre en compte le caractère «feuilleté » du récit du développement d'une spécialité comme l'ICS. Chacun de ses niveaux renvoie à différentes configurations relationnelles (et temporalités), aux dynamiques propres. Dans le cas de l'ICS, l'étude de ces dynamiques montre qu'il faut tempérer la rhétorique de la fluidité et de «l'effacement des frontières » souvent associée à la description des réseaux scientifiques comme «tissus sans coutures » (voir Shinn et Ragouet, 2005). L'analyse des formes de l'internationalité de l'ICS montre en particulier que la compréhension du processus de "globalisation»scientifique passe par la saisie des arrangements, mais aussi de la concurrence pouvant exister entre différentes communautés nationales. Dans ce tableau, les frontières comptent, comme lieux de passages et de frictions. La fidélité à la formule initiale du "tissu sans coutures » de $\mathrm{T}$. Hughes doit en effet inciter à le considérer comme le résultat d'un travail

34. C'est un résultat classique en STS, au moins depuis les années 1970 (voir Dubois, 1999). Et ce d'autant plus que l'étude porte sur une forme de big science, qui donne une grande visibilité à l'encastrement social et à la diversité des « ressources » sur lesquelles s'appuient les scientifiques (Galison et Hevly, 1992, p. 17, p. 356-357). 
collectif - et non comme le plan de travail des acteurs (Gingras, 1995, p. 123-124). Autrement dit, même une «histoire totale [au sens braudélien] de l'activité scientifique » passe par «l'intégration et non la négation » de la pluralité des acteurs et des temporalités (ibid., p. 134-135).

Dans cette perspective, on peut suivre Andrew Abbott (2001, p. 194) et considérer que, si le schéma de Braudel offre bien « un point de départ », le principe de l'emboîtement hiérarchique des temporalités qui l'anime, accordant la primeur à la longue durée, doit être abandonné, pour ouvrir le chantier d'une théorie des liens entre niveaux. Aucun niveau ne «commande » l'autre, a priori : ces liens sont en effet changeants et justiciables d'une enquête empirique portant sur l'articulation des « lignages » d'activité et leurs conséquences. Il s'agit dès lors non seulement d'identifier les processus aux rythmes différents qui informent les entités sociales, mais aussi de penser comment ces processus peuvent se « conditionner mutuellement » (Abbott, 1991, p. 224-225. Voir l'exemple de l'École de Chicago dans Abbott, 1999, ainsi que Jouvenet, 2016). Ainsi, pour le cas du développement de l'ICS, il faut distinguer la dynamique propre à chaque niveau, mais aussi l'impact toujours possible d'un niveau sur les autres - en se demandant, par exemple, comment les accords passés au niveau intergouvernemental (comme le Traité de l'Antarctique) peuvent orienter la coopération internationale entre scientifiques, ou encore ce qui permet aux analyses produites à partir des $I C$ prélevés dans les inlandsis polaires de contribuer à transformer la nature des pôles. La pérennité de cette spécialité dans le paysage scientifique s'explique en effet par un imprévisible retournement (voir infra " L'ICS et le "régime climatique" ») : alors qu'à la fin des années 1950 la présence des scientifiques en Antarctique et au Groenland dépend surtout des enjeux politiques de la Guerre froide, leur travail sur place va conduire à la production de connaissances qui vont s'avérer décisives dans l'émergence du « régime climatique » qui détermine aujourd'hui, en grande partie, les projections internationales sur ces territoires. Le « récit structuré », à la Galison, des contraintes contextuelles orientant le développement international d'un domaine scientifique $^{35}$, apparaît ici insuffisant pour saisir sa force : ce compte rendu est en effet entremêlé à un autre récit, qui relate la façon dont les scientifiques ont exploité les ressources organisationnelles et les prises naturelles à leur disposition pour gagner en crédit et donc en autonomie ${ }^{36}$. Ce cas montre également qu'il faut suivre David Stump (1996, p. 447-449) quand il identifie l'étude de la réciprocité des influences entre les pratiques scientifiques et leur «contexte social» comme une piste susceptible de renouveler la réflexion des STS sur la contextualisation. Si l'arrangement des raisons politiques et scientifiques est une constante de l'internationalité de ce domaine scientifique, son sens a bien évolué. Cet arrangement est le résultat d'un travail à la frontière

35. «Galison veut saisir [...] les contraintes [impositions] pesant sur la connaissance et l'action des scientifiques à travers le récit très structuré établissant comment différents projets, techniques, collectifs et investissements à long terme en capitaux (aussi bien intellectuels que matériels) déterminent l'organisation de la recherche et de ce fait contraignent les types mêmes de choses qui peuvent être découvertes » par les scientifiques (Hacking, 1995, p. 6). Ce récit est structuré de deux manières : d'une part, ces contraintes ont une histoire et, d'autre part, elles agissent suivant des temporalités distinctes, que Peter Galison formule à partir du modèle braudélien (Galison, 1995, p. 15-18).

36. De ce point de vue, l'un des aspects de la dispute entre P. Galison et Andrew Pickering (1995), qui prône le rejet du vocabulaire de la «contrainte » au profit de celui de la « ressource » pour saisir l'historicité des pratiques scientifiques, parait superficiel. Comme l'indiquent les remarques d'Yves Gingras (1995) et Ian Hacking (1995), les deux postures peuvent se compléter plutôt que s'opposer (notamment parce qu'elles renvoient à des niveaux d'analyse différents, plus « micro » pour A. Pickering : son univers descriptif est celui de l'expérimentateur composant avec les « résistances » et « ressources » de son dispositif matériel dans la «temporalité de la pratique »). 
(et des frontières) qui reste à éclairer davantage, afin de mieux restituer les tenants et aboutissants du croisement des « lignages » d'interactions (au sens d'A. Abbott) qui scandent le développement de l'ICS.

\section{Morgan Jouvenet}

Laboratoire Printemps CNRS-Université de Versailles Saint-Quentin-en-Yvelines UFR des Sciences sociales 47 , boulevard Vauban 78047 Guyancourt cedex morgan.jouvenet@uvsq.fr

\section{RÉFÉRENCES BIBLIOGRAPHIQUES}

Аввотт A., 1991, «History and Sociology: The Lost Synthesis », Social Science History, 15, 2, p. 201-238.

Аввотт A., 1999, Department and Discipline. Chicago Sociology at One Hundred, Chicago (IL), The University of Chicago Press.

Аввотт A., 2001, Time Matters. On Theory and Method, Chicago (IL), The University of Chicago Press.

Alley R. B., [2000] 2014, The Two-Mile Time Machine. Ice Cores, Abrupt Climate Change, and Our Future, Princeton (NJ), Princeton University Press.

Aykut S., Dahan A., 2014, Gouverner le climat? 20 ans de négociations internationales, Paris, Presses de Sciences Po.

Belanger D. O., 2006, Deep Freeze. The United States, The International Geophysical Year, and the Origins of Antarctica's Age of Science, Boulder (Co), University Press of Colorado.

Bonneuil C., Fressoz J.-B., 2013, L'événement anthropocène. La Terre, l'histoire et nous, Paris, Le Seuil.

CASSIER M., 1998, «Le partage des connaissances dans les réseaux scientifiques: l'invention de règles de "bonne conduite" par les chercheurs », Revue française de sociologie, 39, 4, p. 701-720.

Crawford E., Shinn T., Sörlin S. (eds.), 1993, Denationalizing Science. The Contexts of International Scientific Practice, Dordrecht, Springer.

Cronon W., 1991, Nature's Metropolis. Chicago and the Great West, New York (NY), Norton.

DansgaARD W., 2005, Frozen Annals. Greenland Ice Cap Research, Copenhagen, Niels Bohr Institute.

Doel R. E., 1997, « The Earth Sciences and Geophysics » dans J. Krige, D. Pestre (eds.), Science in the Twentieth Century, London, Harwood Academic Publishers, p. 361-388.

Doel R. E., 2003, « Constituting the Postwar Earth Sciences: The Military's Influence on the Environmental Sciences in the USA After 1945 », Social Studies of Science, 33,5, p. 635-666. 
Doel R. E., WrÅKberg U., Zeller S., 2014, « Science, Environment, and the New Arctic », Journal of Historical Geography, 44, p. 2-14.

DuboIs M., 1999, Introduction à la sociologie des sciences et des connaissances scientifiques, Paris, Presses universitaires de France.

Edwards P. N., 2010, A Vast Machine. Computer Models, Climate Data, and the Politics of Global Warming, Cambridge (MA), The MIT Press.

Elzinga A., 1993, « Antarctica: The Construction of a Continent by and for Science » dans E. Crawford, T. Shinn, S. Sörlin (eds.), Denationalizing Science. The Contexts of International Scientific Practice, Dordrecht, Springer, p. 73-106.

FogG G. E., 1992, A History of Antarctic Science, Cambridge, Cambridge University Press.

Forman P., 1987, «Behind Quantum Electronics: National Security as Basis for Physical Research in the United States, 1940-1960», Historical Studies in the Physical and Biological Sciences, 18, 1, p. 149-229.

Frezzotti M., 2011, «Core of Ice », Nature Geoscience, 68, 4, p. 68.

Galison P., 1995, «Context and Constraints » dans J. Z. Buchwald (ed.), Scientific Practice. Theories and Stories of Doing Physics, Chicago (IL), The University of Chicago Press, p. 13-41.

Galison P., 2008, « Ten Problems in History and Philosophy of Science », Isis, 99, p. 111-124.

Galison P., Hevly B. (eds.), 1992, Big Science: The Growth of Large-Scale Research, Stanford (CA), Stanford University Press.

Galison P., Stump D. J. (eds.), 1996, The Disunity of Science: Boundaries, Contexts, and Power, Stanford (CA), Stanford University Press.

Gingras Y., 1995, «Following Scientists through Society? Yes, but at Arm's Length! » dans J. Z. Buchwald (ed.), Scientific Practice. Theories and Stories of Doing Physics, Chicago (IL), The University of Chicago Press, p. 123-148.

Gingras Y., 2010, Propos sur les sciences. Entretiens avec Yanick Villedieu, Paris, Raisons d'agir.

Gingras Y., 2013, Sociologie des sciences, Paris, Presses universitaires de France.

GrevsmüHL S. V., 2014, La terre vue d'en haut. L'invention de l'environnement global, Paris, Le Seuil.

GrevsmüHL S. V., 2016, «Laboratory Metaphors in Antarctic History: From Nature to Space » dans C. Kehrt, F. Torma (eds.), Exploring Ice and Snow in the Cold War, Oxford, Berghahn Books [à paraître].

Grove R. H., [1993] 2013, Les îles du Paradis. L'invention de l'écologie aux colonies, 1660-1854, Paris, La Découverte.

Hacking G. I., 1995, « Introduction » dans J. Z. Buchwald (ed.), Scientific Practice. Theories and Stories of Doing Physics, Chicago (IL), The University of Chicago Press, p. 1-9.

Hauglustaine D., Jouzel J., Masson-Delmotte V. (dir.), 2008, Atmosphère, atmosphère, Paris, Le Pommier/CNAM.

Hecht G., [1998] 2004, Le rayonnement de la France : énergie nucléaire et identité nationale après la Seconde Guerre mondiale, Paris, La Découverte.

Hevly B., 1996, « The Heroic Science of Glacier Motion », Osiris, 11, p. 66-86. 
Howkins A., 2010, « Science, Environment, and Sovereignty: The International Geophysical Year in the Antarctic Peninsula Region» dans R. D. LAUniUs, J. R. Fleging, D. H. Devorkin (eds.), Globalizing Polar Science. Reconsidering the International Polar and Geophysical Years, New York (NY), Palgrave MacMillan, p. 245-264.

Howkins A., 2011, «Melting Empires? Climate Change and Politics in Antarctica since the International Geophysical Year », Osiris, 26, p. 180-197.

Hughes E., 1976, « The Social Drama of Work », Mid-American Review of Sociology, 1,1, p. 1-7.

INGOLD A., 2011, «Écrire la nature. De l'histoire sociale à la question environnementale ?», Annales. Histoire, Sciences sociales, 66, 1, p. 1-29.

Jouvenet M., 2016, «Contextes et temporalités dans la "sociologie processuelle" d'Andrew Abbott », Annales. Histoire, Sciences sociales, 71, 3 [à paraître]

Jouzel J., 2013, «A Brief History of Ice Core Science Over the Last 50 Years », Climate of the Past, 9, p. 2525-2547.

Jouzel J., Debroise A., 2007, Le climat : jeu dangereux. Dernières nouvelles de la planète, Paris, Dunod.

Jouzel J., Lorius C., Raynaud D., 2008, Planète blanche. Les glaces, le climat et l'environnement, Paris, Odile Jacob.

JugIE G., 2007, « La station franco-italienne Concordia (entretien) » dans L. MAYET (éd.), L'aventure polaire, Paris, Le Pommier, p. 37-47.

KoHLER R. E., 1994, Lords of the Fly. Drosophilia Genetics and the Experimental Life, Chicago (IL), The University of Chicago Press.

KOHLER R. E., 2002, Landscapes and Labscapes: Exploring the Lab-Field Border in Biology, Chicago (IL), The University of Chicago Press.

KoHLER R. E., 2011, «History of Field Science. Trends and Prospects » dans J. Vetter (ed.), Knowing Global Environments. New Historical Perspectives on the Field Sciences, New Brunswick (NJ), Rutgers University Press, p. 212-240.

Krige J., 2014a, « Concluding Remarks » dans N. Oreskes, J. KrIGE (eds.), Science and Technology in the Global Cold War, Cambridge (MA), The MIT Press, p. 431-441.

KRIGE J., 2014b, «Embedding the National in the Global: Us-French Relationships in Space Science and Rocketry in the 1960s » dans N. ORESKES, J. KRIGE (eds.), Science and Technology in the Global Cold War, Cambridge (MA), The MIT Press, p. 227-250.

LATOUR B., 1989, La science en action, Paris, Gallimard.

LAtour B., 2008, «Pour un dialogue entre science politique et science studies », Revue française de science politique, 58, 4, p. 657-678.

Launius R. D., Fleming J. R., Devorkin D. H. (eds.), 2010, Globalizing Polar Science. Reconsidering the International Polar and Geophysical Years, New York (NY), Palgrave MacMillan.

LePETIT B., 1996, «De l'échelle en histoire » dans J. Revel (éd.), Jeux d'échelles. La microanalyse à l'expérience, Paris, Gallimard/Le Seuil, p. 71-94.

LEWIS R. S., 1965, A Continent for Science. The Antarctic Adventure, London, Secker \& Warburg.

Lewis R. S., Smith P. M. (eds.), 1973, Frozen Future. A Prophetic Report from Antarctica, New York (NY), Quadrangle Books. 
LoRIUS C., 1993, Glaces de l'Antarctique. Une mémoire, des passions, Paris, Le Seuil.

Lorius C., Carpentier L., 2010, Voyage dans l'anthropocène, Arles, Actes Sud.

Mallard G., Paradeise C., Peerbaye A. (eds.), 2009, Global Science and National Sovereignty, New York (NY), Routledge.

Martin-Nielsen J., 2013, " "The Deepest and Most Rewarding Hole Ever Drilled": Ice Cores and the Cold War in Greenland », Annals of Science, 70, 1, p. 47-70.

Mayewski P. A., White F., 2002, The Ice Chronicles. The Quest to Understand Global Climate Change, Hanover, University Press of New England.

MCNEILl J. R., 2010, Du nouveau sous le soleil. Une histoire de l'environnement mondial au XXe siècle, Paris, Champ Vallon.

Naylor S., Dean K., Siegert M., Turchetti S., 2008, « Science, Geopolitics and the Governance of Antarctica », Nature Geoscience, 1, p. 143-145.

Nye J., 2004, Soft Power. The Means to Success in World Politics, New York (NY), Public Affairs.

Oreskes N., 2004, «Beyond the Ivory Tower: The Scientific Consensus on Climate Change », Science, 306, 5702, p. 1686.

Oreskes N., 2014a, « Changing the Mission: From the Cold War to Climate Change » dans N. Oreskes, J. Krige (eds.), Science and Technology in the Global Cold War, Cambridge (MA), The MIT Press, p. 141-187.

Oreskes N., 2014b, «Introduction » dans N. Oreskes, J. Krige (eds.), Science and Technology in the Global Cold War, Cambridge (MA), The MIT Press, p. 1-9.

Oreskes N., Krige J. (eds.), 2014, Science and Technology in the Global Cold War, Cambridge (MA), The MIT Press.

Pestre D., 2010, « Dix thèses sur les sciences, la recherche scientifique et le monde social, 1945-2010», Le mouvement social, 233, p. 13-29.

Pestre D., 2012, «Debates in Trans-National and Science Studies », The British Journal for the History of Science, 45, 3, p. 425-442.

Petit J.-R., 2013, Vostok. Le dernier secret de l'Antarctique, Paris, Paulsen.

PiCKering A., 1995, «Beyond Constraint: The Temporality of Practice and the Historicity of Knowledge » dans J. Z. Buchwald (ed.), Scientific Practice. Theories and Stories of Doing Physics, Chicago (IL), The University of Chicago Press, p. $42-55$.

Pyne S. J., [1987] 2004, The Ice, London, Phoenix.

Ravetz J., 2006, The No-Nonsense Guide to Science, Oxford, New Internationalist.

Raynaud D., Chappellaz J., 2008, « Des gaz dans la glace » dans D. Hauglustaine, J. Jouzel, V. Masson-Delmotte (eds.), Atmosphère, atmosphère, Paris, Le Pommier/CNAM, p. 117-137.

Revel J., 1996, « Microanalyse et construction du social » dans J. Revel (éd.), Jeux d'échelles. La microanalyse à l'expérience, Paris, Gallimard/Le Seuil.

Rosental P.-A., 1996, "Construire le "macro" par le "micro" : Fredrik Barth et la microstoria » dans J. ReVEL (éd.), Jeux d'échelles. La microanalyse à l'expérience, Paris, Gallimard/Le Seuil, p. 141-159.

RuDwick M., 1996, «Geological Travel and Theoretical Innovation: The Role of "Liminal" Experience », Social Studies of Science, 26, 1, p. 143-159. 
ShaPIN S., 1992, « Discipline and Bounding: The History and Sociology of Science as Seen Through the Externalism-Internalism Debate », History of Science, 30, p. 333-369.

Shinn T., Ragouet P., 2005, Controverses sur la science. Pour une sociologie transversaliste de l'activité scientifique, Paris, Raisons d'agir.

SöRLIN S., 1993, «National and International Aspects of Crossboundary Science: Scientific Travel in the 18th Century » dans E. Crawford, T. ShInN, S. SörLIN (eds.), Denationalizing Science. The Contexts of International Scientific Practice, Dordrecht, Springer, p. 43-72.

StumP D. J., 1996, «Afterword: New Directions in the Philosophy of Science Studies » dans P. Galison, D. Stump (eds.), The Disunity of Science: Boundaries, Contexts, and Power, Stanford (CA), Stanford University Press, p. 443-450.

Traweek S., 1988, Beamtimes and Lifetimes. The World of High Energy Physicists, Cambridge (MA), Harvard University Press.

Traweek S., 1992, «Big Science and Colonialist Discourse: Building High-Energy Physics in Japan » dans P. Galison, B. Hevly (eds.), Big Science: The Growth of Large-Scale Research, Stanford (CA), Stanford University Press, p. 100-128.

Turchetti S., Herran N., Boudia S., 2012, « Have We Ever Been Transnational? Towards a History of Science Across and Beyond Borders », British Journal for the History of Science, 45, 3, p. 319-336.

Turchetti S., Naylor S., Dean K., Siegert M., 2008, « On Thick Ice: Scientific Internationalism and Antarctic Affairs, 1957-1980», History and Technology, 24, 4, p. 351-376.

Turchetti S., Roberts P., 2014a, «Introduction. Knowing the Enemy, Knowing the Earth » dans S. TURChETTI, P. ROBERTS (eds.), The Surveillance Imperative Geosciences during the Cold War and Beyond, New York (NY), Palgrave MacMillan, p. 1-19.

TurchetTi S., Roberts P. (eds.), 2014b, The Surveillance Imperative Geosciences during the Cold War and Beyond, New York (NY), Palgrave MacMillan.

Van Damme S., 2014, «Laborieuse nature. Penser le travail des sciences exactes avec Simon Schaffer», La vie des idées, 27 mai 2014: http://www.laviedes idees.fr/Laborieuse-Nature.html.

VeTter J., 2011, «Introduction» dans J. VeTtER (ed.), Knowing Global Environments. New Historical Perspectives on the Field Sciences, New Brunswick (NJ), Rutgers University Press, p. 1-16.

Walton D. W. H. (ed.), 2013, Antarctica: Global Science from a Frozen Continent, Cambridge, Cambridge University Press.

WeART S. R., 2008, The Discovery of Global Warming. Revised and Expanded Edition, Cambridge (MA), Harvard University Press.

WeIss C., 2015, « How Do Science and Technology Affect International Affairs? », Minerva, 53, 4, p. 411-430.

White R., 1996, «"Are You an Environmentalist or Do You Work for a Living?”: Work and Nature » dans W. CRONON (ed.), Uncommon Ground: Rethinking the Human Place in Nature, New York (NY), W. W. Norton \& Co, p. 171-185.

WitzE A., 2015, « Super-Fast Antarctic Drills Ready to Hunt for Oldest Ice », Nature, 526,7575 , p. 618-619. 
From the poles to the laboratories: stages and scales of international cooperation in paleoclimatology (1955-2015)

Ice Core Science (ICS) is a paleoclimatology specialty aiming to reconstruct the dynamics of past climates, from laboratory analysis of ice samples drilled in Greenland and Antarctica. The field idiosyncrasies and the geopolitical stakes attached to these polar territories explain why this specialty has been characterized, from its beginnings, by strong international cooperation. This article relates the meaning and forms of this cooperation, focusing on the organization of field work by scientists, and presenting their coordination in the global network enabling the flow of brittle climate archives from the field to the laboratories. The historical analysis reveals a strong initial influence of the Cold War on international cooperation in ICS. But it also shows how the ICS community has consolidated its presence and credibility beyond this context, changing the polar environment's very nature in the course of the global warming debate. Finally, the many faces of Ics's internationalism provide an empirical fulcrum for the renewal of the discussion about the treatment of context in science studies, as the conclusion of the paper argues.

Key words. Science studies - Paleoclimatology - Cooperation - Transnational STUDIES - GLOBALIZATION

\section{ZUSAMMENFASSUNG}

\section{Von den Polen zu den Labors: die Stufen der internationalen Zusammenarbeit in der Paläoklimatologie (1955-2015)}

Die Ice Core Science (ICS) ist eine Spezialität der Paläoklimatologie mit der die Dynamik der Klimas in der Vergangenheit nachvollzogen wird, ausgehend von der Laboranalyse von Eisstücken aus Bohrungen in Grönland und in der Antarktis. Die Eigenheiten des Polarbodens und die mit diesen Territorien verbundenen geopolitischen Herausforderungen erklären, daß die ICS sich von Beginn an durch eine weitgehende internationale Zusammenarbeit auszeichnet. Der Aufsatz berichtet über den Sinn und die Formen dieser Zusammenarbeit indem er die Organisation der Feldkampagnen durch die Wissenschaftler mit Interesse betrachtet und ihre Koordinierung innerhalb eines Mobilisierungsnetzes des empfindlichen Klimaarchivs vorstellt -eine Globalisierungsinfrastruktur, in der die Pole mit den Labors verbunden sind. Die Analyse der Entwicklung der ICS zeigt den anfänglichen Einfluß des Kontexts des Kalten Kriegs auf die internationale wissenschaftliche Zusammenarbeit. Sie zeigt weiterhin, wie diese Spezialität über diesen Kontext hinaus ihre Aktivität verstetigen konnte indem sie die Natur der Pole verändert und zur Debatte über die Klimaerwärmung beigetragen hat. Der Ausbruch des „internationalen Sachverhalts“ auf diesem Gebiet stellt einen empirischen Stützpunkt dar, der die Überlegungen zur Behandlung des Kontexts in den Science Studies nährt, und das Fazit des Artikels bringt Elemente in diesem Sinn.

Schlagwörter. WisSENSCHAFTSSTUdien - PALÄOKLIMATOlOGIE - ZUSAMMENARBEIT Transnationale Studien - Globalisierung 
Resumen

De los polos a los laboratorios: escalas de la cooperación internacional en paleoclimatología (1955-2015)

La Ice Core Science (ICS) es una rama de la paleoclimatología que permite reconstituir los climas del pasado, basándose en el análisis en laboratorio de trozos de hielo extraídos de los suelos de Groenlandia y de la Antártida. Las particularidades del suelo polar y las problemáticas geopolíticas asociadas con estos territorios explican que la ICS se caracterice, ya desde su aparición, por una fuerte cooperación internacional. En este artículo se explicitan el sentido y las formas de esta cooperación, mediante la observación de la organización de los estudios de campo por los científicos, y la presentación de su coordinación dentro de la red de movilización de los escasos archivos climáticos - una infraestructura de globalización que une los polos con los laboratorios. El análisis del desarrollo de la ıcs revela la influencia inicial del contexto de la Guerra Fría sobre la cooperación científica internacional. Muestra también cómo esta especialidad perennizó su actividad más allá de ese contexto, transformando la naturaleza de los polos y contribuyendo en los debates en torno al calentamiento global. La dispersión del "hecho internacional", en este campo, constituye una piedra de toque empírica que alimenta la reflexión sobre la relación con el contexto en los Science Studies; en la conclusión de este artículo aportamos elementos en este sentido.

Palabras claves. Estudios de las Ciencias - PALeOClimatología - CoOperación ESTUDIOS TRANSNACIONALES - GLOBALIZACIÓN 